\title{
Clinical Efficacy of Polymyxin B in Patients Infected with Carbapenem-Resistant Organisms
}

\author{
Qiong Lu (iD ${ }^{1, *}$ \\ Guo-Hua $\mathrm{Li}^{1}{ }^{1} *$ \\ Qiang $\mathrm{Qu}^{2}$ \\ Hai-Hong Zhu' \\ Yue Luo ${ }^{3}$ \\ Han Yan' \\ Hai-Yan Yuan' \\ Jian $Q u^{\prime}$
}

'Department of Pharmacy, the Second Xiangya Hospital, Central South University; Institute of Clinical Pharmacy, Central South University, Changsha, People's Republic of China; ${ }^{2}$ Department of Pharmacy, Xiangya Hospital, Central South University, Changsha, People's Republic of China; ${ }^{3}$ Department of Pharmacy, the People's Hospital of LIUYANG, Liuyang, People's Republic of China

*These authors contributed equally to this work
Correspondence: Jian $\mathrm{Qu}$

Department of Pharmacy, the Second Xiangya Hospital, Central South

University; Institute of Clinical Pharmacy,

Central South University, No. 139 Middle

Renmin Road, Changsha, People's

Republic of China

Tel +86-|5973|906/4

Fax +86-073I-85292072

Email qujianstanley@csu.edu.cn
Purpose: Carbapenem-resistant organisms (CROs) pose great challenges for clinical treatment. Polymyxin B (PMB) is one of the "last resort" choices of CRO infections. We explored the possible factors affecting PMB efficacy.

Patients and Methods: This retrospective study involved CRO-infected patients treated with $\mathrm{PMB}$ for $\geq 72 \mathrm{~h}$. The endpoint indicator was clinical efficacy. We compared the characteristics (demographics, pathogenic bacteria, PMB treatment) between patients who had "clinical success" (CS) and "clinical failure" (CF).

Results: A total of 191 patients were enrolled: 110 in the CS group and 81 in the CF group. The total cumulative dose for the CS group was higher than the CF group [1100 (700-$1443.75)$ vs $800(500-1112.5) \mathrm{mg} ; P=0.001]$. Treatment duration in the CS group was longer than the CF group [11 (8-14) vs $8(6-11)$ days; $P<0.000]$. Multivariate logistic regression analysis showed mechanical ventilation, vasoactive agents, multiple-site infection, and total cumulative dose to be independently associated with clinical efficacy. Cox survival analysis for 30-day mortality also showed that the use of vasoactive agents and the total cumulative dose of PMB could influence survival time and mortality rate independently.

Conclusion: PMB had good efficacy and a low prevalence of adverse reactions. The total cumulative dose, duration of PMB treatment, mechanical ventilation, vasoactive agents, and multiple-site infection were factors associated with the clinical efficacy of PMB.

Keywords: polymyxin B, carbapenem-resistant organisms, clinical efficacy, adverse effect, cumulative dose

\section{Introduction}

Carbapenem-resistant organisms (CROs) are Gram-negative bacteria that are resistant to carbapenem antibiotics. The main species of CROs are Enterobacteralesresistant organisms (CREs), Acinetobacter baumannii-resistant organisms, and Pseudomonas aeruginosa-resistant organisms. ${ }^{1}$ Due to limited treatment options and frequently a poor prognosis, CROs pose considerable challenges for clinical treatment. Therefore, research on the treatment of CRO infections has attracted widespread attention. Drugs reported to be efficacious in treating CROs include colistin, aminoglycosides, tigecycline, fosfomycin, ceftazidime/avibactam, and ceftolozane/tazobactam, and combination therapy can be superior to monotherapy. ${ }^{2}$

Polymyxin B (PMB) is a type of polypeptide antibiotic discovered in $1947 .^{3}$ Polymyxins have strong antibacterial activity against most Gram-negative bacteria, but their clinical use has been restricted due to side effects, such as neurotoxicity and nephrotoxicity. Along with the emergence of multidrug-resistant Gram-negative bacteria, PMB and colistin have attained renewed attention because of their specific 
effects. Studies have shown that PMB has lower nephrotoxicity compared with colistin at recommended doses. ${ }^{4-6}$ Therefore, PMB has been used as a first-line agent for CRO infections since it was listed in mainland China.

Studies on the pharmacokinetics, pharmacodynamics, and medications of PMB have been deepening gradually. ${ }^{7}$ Related studies have demonstrated the efficacy, toxicity, and efficacy prediction of PMB in the treatment of CRO infections. Due to the synergistic effect in in vitro studies, colistin or PMB are usually combined with carbapenems or tigecycline. ${ }^{8}$ Early treatment with PMB and sound renal function usually lead to better outcomes. ${ }^{9,10}$ However, PMB may have an inferior microbiologic clearance rate of carbapenem-resistant Klebsiella pneumoniae (CRKP) compared with aminoglycoside. ${ }^{11}$

Although PMB is used widely clinically, several factors affecting efficacy and side effects must be explored in realworld scenarios. Therefore, we conducted a retrospective study on the administration, factors affecting efficacy, and adverse effects of PMB to provide a reference for the rational use of PMB.

\section{Patients and Methods}

\section{Ethical Approval of the Study Protocol}

The study protocol was approved by the Ethics Committees of the Second Xiangya Hospital of Central South University (LYF-2020021) in Changsha, China. It was carried out by the ethical standards laid down in the 1964 Declaration of Helsinki and its later amendments. Patients gave their written informed consent to have their data included in this study.

\section{Patients}

The Second Xiangya Hospital of Central South University is a large-scale comprehensive first-class hospital, covering an area of more than 260 acres, with 3500 beds and 40 clinical departments. A study conducted by Xiangya Hospital of Central South University in Hunan Province in 2013 showed that the resistance rates of Acinetobacter baumannii to imipenem and meropenem were $49.4 \%$ and $48.3 \%$ respectively. ${ }^{12}$ Our retrospective study involved patients admitted to the Second Xiangya Hospital from 2018 to 2019. The inclusion criteria were patients: (i) with a medication history of PMB (Shanghai Number 1 Biochemical \& Pharmaceuticals, Shanghai, China); (ii) infected with a CRO according to twice bacterial-culture results at least; (iii) with complete medical data and basic information. The exclusion criteria were: (i)
PMB administration $<72 \mathrm{~h}$; (ii) patients with severe liver/ kidney dysfunction or a malignant tumor before PMB treatment; (iii) patients without an intravenous drip. After selecting patients based on inclusion and exclusion criteria, patients were divided into two groups according to treatment results.

\section{Collection of Clinical Data}

The collection of clinical data was based on medical records. We collected data on basic demographic characteristics; diagnoses; infection sites; pathogenic bacteria (and their sensitivity); Acute Physiology and Chronic Health Evaluation (APACHE) II score; medication regimens (PMB and drugs it was combined with); treatment duration; parameters of efficacy evaluation; treatment results; adverse effects.

\section{Definitions}

Patients were divided into "clinical success (CS)" and "clinical failure (CF)" groups according to efficacy. "CS" was defined as improvements of microbiologic and clinical symptoms and parameters including body temperature, APACHE II score, biochemistry indicators of infection (White blood cell count in adults $\leq 10^{9}$, C-reactive protein $\leq 10 \mathrm{mg} / \mathrm{L}$, Procalcitonin $<$ $0.05 \mathrm{ng} / \mathrm{mL}$, erythrocyte sedimentation rate $<15 \mathrm{~mm} / \mathrm{h}$ ), twice negative culture results at least, control of infection symptoms and clinician documented improvements at the end of treatment. "CF" was defined as failure to meet all the criteria for $\mathrm{CS}$ and deterioration/persistence of infection symptoms or death. ${ }^{10,13,14}$ "Adverse effects" were defined as harmful reactions to PMB unrelated to the purpose of treatment which emerged after using the drug and disappeared when its use was stopped, including nerve-muscle blockade, nephrotoxicity, or skin pigmentation. Nephrotoxicity was based on the risk, injury, failure, loss of kidney function, and end-stage kidney disease (RIFLE) criteria. ${ }^{15}$

\section{Microbiology}

Types of bacteria and minimum inhibitory concentrations (MICs) were determined by a VITEK $^{\circledR} 2$ system (bioMérieux, Marcy-l'Étoile, France) based on recommendations of the Clinical and Laboratory Standards Institute. Testing for colistin sensitivity was done by laboratory physicians using analytical instruments by the brothmicrodilution method. "Carbapenem resistance" was defined as the MIC of imipenem and meropenem $\geq 4 \mathrm{mg} /$ L. According to the European Committee on Antimicrobial Susceptibility Testing (EUCAST, v8.0, 2018), bacteria were resistant to colistin if their MICs were $>2 \mathrm{mg} / \mathrm{L}^{16}$ 


\section{Statistical Analyses}

Statistical analyses were undertaken using SPSS v21.0 (IBM, Armonk, NY, USA). Count data were analyzed by the chi-square test. Data with a normal distribution are expressed as the mean \pm standard deviation, and were analyzed by the independent $t$-test. Data with a nonnormal distribution are expressed by the median value and interquartile range and were analyzed by the nonparametric test. Multivariate logistic regression was used to assess for the independent predictors of the clinical efficacy of PMB. Factors entered into the multivariate logistic model included any baseline differences between CS and $\mathrm{CF}$ groups that had $\mathrm{P}<0.1$ on bivariate analysis. $\mathrm{P}<0.05$ was considered significant. Moreover, those variables were included in the Cox model for 30-day mortality, $\mathrm{P}<0.05$ was considered significant.

\section{Results}

\section{Characteristics}

A total of 273 patients administered PMB were found in the database of the Second Xiangya Hospital of Central South University between 2018 and 2019. According to the inclusion and exclusion criteria, 191 patients were included in statistical analyses and most patients came from the "Intensive Care Unit" (n, 114; 59.7\%).

The overall information for patients, including basic information, diagnostic information, and microbial infections, are listed in Table 1. Among all patients, $61.3 \%$ (n, 117) had a single-site infection and, in $89.0 \%$ (n, 170) of cases, the infection site was the respiratory tract. Also, 210 CRO strains were isolated from 191 patients. The highest proportion of pathogenic bacteria was Acinetobacter baumannii (n, 121; 63.4\%), followed by Klebsiella pneumoniae (n, 49; 25.6\%), Pseudomonas aeruginosa (n, 33; 17.2\%), Escherichia coli and Enterobacter cloacae (n, 7; 3.7\%).

\section{Medications and Outcomes}

All patients used PMB (Shanghai Number 1 Biochemical \& Pharmaceuticals) according to manufacturer instructions and International Consensus Guidelines for the Optimal Use of the Polymyxins. ${ }^{17}$ All patients included in statistical analyses were administered PMB by intravenous drip (n, 191; 100\%) (Table 1). We found that $36.1 \%(n, 69)$ of patients were given a loading dose of 50.0-100.0 mg. The dose per kilogram (mg/kg/q12h) was 0.86 (range, 0.82-1.00). Treatment duration was 10.0 (range, 7.0-13.5) days. PMB combined with another drug was given to $91.1 \%(\mathrm{n}, 180)$ of cases.
Meropenem was the agent combined most commonly with PMB (n, 64; 33.5\%), followed by tigecycline (n, 63; 33.0\%), and cefoperazone/sulbactam ( $\mathrm{n}, 53 ; 27.7 \%)$.

There were 110 patients in the CS (57.6\%) group and 81 (42.4\%) patients in the CF group. The 30-day mortality rate was $15.2 \%(\mathrm{n}, 29)$ and the 14-day mortality rate was $12.6 \%$ (n, 24). The median mortality time was $9.0(6.0$ 12.8 ) days. Bacteria elimination was successful in $24.0 \%$ $(n, 46)$ of cases, and the time required to clear bacteremia was 8.0 (range, 6.0-13.0) days. Duration of hospitalization was 34.0 (range, 21.0-56.8) days (Table 2).

\section{Factors Related to the Clinical Efficacy of PMB}

To explore the factors that affected the clinical efficacy of PMB, we compared all the demographics, clinical characteristics, and details of PMB treatment (dose, loading dose, timing of medication, combined medication, treatment duration) between the two groups. There was no significant difference in the time for starting PMB treatment after CROs infection confirmation (1.0 vs 1.5 days, $\mathrm{P}=0.761)$. Meanwhile, statistical difference in the proportion of common combination drugs did not exist in $\mathrm{CF}$ and $\mathrm{CS}$ groups (Table 1). The median total cumulative dose of patients in the CS group tended to be higher $(1100$ vs $800 \mathrm{mg}, \mathrm{P}=$ 0.001 ) and the median treatment duration was longer (11 vs 8 days, $\mathrm{P}=0.000$ ). More patients in the $\mathrm{CF}$ group had mechanical ventilation and vasoactive agents $(\mathrm{P} \leq 0.001)$. The clinical effect for patients with multiple-site infections was worse compared with that for patients with a single-site infection $(29.1 \%$ vs $70.9 \%, \mathrm{P}=0.002)$ (Table 1$)$. The Mantel-Haenszel chi-square test revealed a linear association between treatment duration ( $\mathrm{P}=0.003)$ and total cumulative dose $(\mathrm{P}=0.005)$, which correlated with CS (Figure 1). The most common combination drugs include meropenem, tigecycline, and Cefoperazone/sulbactam. However, no significant differences were found for the mode of administration and drug combination. In addition, we performed a univariate analysis with 30-day mortality as the endpoint, and obtained results similar to the analysis with clinical efficacy as the endpoint, mechanical ventilation, vasoactive agents, total cumulative dose and treatment duration may influence the efficacy of PMB (Table S1).

We also created a multivariate logistic regression model for the clinical efficacy of PMB that incorporated use of mechanical ventilation and vasoactive agents, multiple-site infection, duration of PMB treatment (days), and total 
Table I Clinical Characteristics of Patients in the CS Group and CF Group After PMB Treatment

\begin{tabular}{|c|c|c|c|c|}
\hline \multirow[t]{2}{*}{ Parameters } & Total & Success & Failure & \multirow[t]{2}{*}{$\mathbf{P}$} \\
\hline & $(N=191)$ & $(N=|| 0)$ & $(N=8 I)$ & \\
\hline Age & $55.0 \pm 20.2$ & $53.3 \pm 18.4$ & $57.3 \pm 22.3$ & 0.187 \\
\hline Sex (male) & I 40 (73.3\%) & $84(76.4 \%)$ & 56 (69.1\%) & 0.213 \\
\hline APACHE II score & $19.0(12.0-24.2)$ & $19.0(\mid 3.2-24.0)$ & $19.0(11.0-26.5)$ & 0.878 \\
\hline Mechanical ventilation & $|3|(68.6 \%)$ & $60(54.5 \%)$ & 71 (87.6\%) & $<0.001$ \\
\hline Vasoactive agents & I0I (52.9\%) & 41 (37.3\%) & 60 (74.1\%) & $<0.001$ \\
\hline \multicolumn{5}{|l|}{ Infection site } \\
\hline Multiple & 74 (38.7\%) & 32 (29.1\%) & 42 (51.9\%) & 0.002 \\
\hline Single & 117 (6I.3\%) & 78 (70.9\%) & 39 (48.1\%) & \\
\hline \multicolumn{5}{|l|}{ Source of infection } \\
\hline Respiratory tract & I 70 (89.0\%) & 100 (90.9\%) & 70 (86.4\%) & 0.233 \\
\hline Blood & 27 (14.1\%) & $12(10.9 \%)$ & $15(18.5 \%)$ & 0.145 \\
\hline Intracranial & $10(5.2 \%)$ & $6(5.5 \%)$ & 4 (4.9\%) & 1.000 \\
\hline Urinary tract & $13(6.8 \%)$ & $7(6.4 \%)$ & $6(7.4 \%)$ & 0.795 \\
\hline Digestive tract & $3(1.6 \%)$ & $2(1.8 \%)$ & $\mathrm{I}(\mathrm{I} .2 \%)$ & 1.000 \\
\hline Abdomen & $23(12.0 \%)$ & $13(6.4 \%)$ & $10(12.3 \%)$ & 0.940 \\
\hline Skin/soft tissue & $3(1.6 \%)$ & $2(1.8 \%)$ & I (I.2\%) & 1.000 \\
\hline \multicolumn{5}{|l|}{ Underlying diseases } \\
\hline Pulmonary disease & 140 (73.3\%) & 79 (7I.8\%) & 61 (75.3\%) & 0.692 \\
\hline Hypertension & $54(28.3 \%)$ & 35 (31.8\%) & 19 (23.4\%) & 0.187 \\
\hline Diabetes mellitus & 27 (14.1\%) & 15 (I3.6\%) & 12 (14.8\%) & 0.844 \\
\hline Recent surgery & 17 (8.9\%) & $12(10.9 \%)$ & $5(6.2 \%)$ & 0.246 \\
\hline Nervous system & $58(30.4 \%)$ & $39(35.5 \%)$ & $19(23.4 \%)$ & 0.067 \\
\hline \multicolumn{5}{|l|}{ Pathogenic bacteria } \\
\hline Acinetobacter baumannii & 121 (63.4\%) & $70(63.6 \%)$ & $51(63.0 \%)$ & 0.838 \\
\hline Klebsiella pneumoniae & $49(25.6 \%)$ & $30(27.3 \%)$ & $19(23.4 \%)$ & 0.519 \\
\hline Pseudomonas aeruginosa & $33(17.2 \%)$ & $18(16.4 \%)$ & $15(18.5 \%)$ & 0.726 \\
\hline Escherichia coli or Enterobacter cloacae & $7(3.7 \%)$ & $2(1.8 \%)$ & $5(6.2 \%)$ & 0.139 \\
\hline Loading dose (N, \%) & $69(36.1 \%)$ & $43(39.1 \%)$ & $26(32.1 \%)$ & 0.292 \\
\hline Loading dose $(\mathrm{mg} / \mathrm{d})$ & $50.0(50.0-100.0)$ & $50.0(50.0-100.0)$ & $50.0(50.0-100.0)$ & 0.477 \\
\hline Maintenance dose $(\mathrm{mg} / \mathrm{d})$ & $50.0(50.0-50.0)$ & $100.0(100.0-100.0)$ & $100.0(100.0-100.0)$ & 0.651 \\
\hline Total cumulative dose (mg) & $950.0(650.0-1350.0)$ & $1100.0(700.0-1443.8)$ & $800.0(500.0-1112.5)$ & 0.001 \\
\hline Dose per kg (mg/kg/ql2h) & $0.86(0.82-1.00)$ & $0.87(0.79-1.00)$ & $0.86(0.83-1.00)$ & 0.541 \\
\hline Daily PMB dose (mg) & $100.0(100.0-100.0)$ & $100.0(100.0-100.0)$ & $100.0(100.0-100.0)$ & 0.794 \\
\hline \multicolumn{5}{|l|}{ Administration } \\
\hline Intravenous drip & 191 (100.0\%) & 110 (100.0\%) & 81 (100.0\%) & 1.000 \\
\hline Intrathecal injection & $5(2.6 \%)$ & $3(2.7 \%)$ & $2(2.5 \%)$ & 1.000 \\
\hline Inhalation & $8(4.2 \%)$ & $6(5.5 \%)$ & $2(2.5 \%)$ & 0.470 \\
\hline Starting PMB treatment after CRO confirmed (days) & I.0 (0.0-4.0) & $1.0(0.0-4.0)$ & $1.5(0.0-3.8)$ & 0.761 \\
\hline Treatment duration (days) & $10.0(7.0-13.5)$ & $11.0(8.0-14.0)$ & $8.0(6.0-11.0)$ & $<0.001$ \\
\hline
\end{tabular}

(Continued) 
Table I (Continued).

\begin{tabular}{|l|c|c|c|c|}
\hline \multirow{2}{*}{ Parameters } & Total & Success & Failure & P \\
\cline { 2 - 5 } & $\mathbf{( N = 1 9 1 )}$ & $\mathbf{( N = 1 1 0 )}$ & $\mathbf{( N = 8 1 )}$ & \\
\hline Combination & & & & \\
PMB alone & $17(8.9 \%)$ & $7(6.4 \%)$ & $(12.3 \%)$ & 0.159 \\
Meropenem & $64(33.5 \%)$ & $40(36.4 \%)$ & $24(29.6 \%)$ & 0.302 \\
Tigecycline & $63(33.0 \%)$ & $32(29.1 \%)$ & $31(38.3 \%)$ & 0.203 \\
\hline Cefoperazone/sulbactam & $53(27.7 \%)$ & $31(28.2 \%)$ & $22(27.2 \%)$ & 0.836 \\
\hline
\end{tabular}

Notes: Data are reported as numbers (\%), mean \pm standard deviation or median values (interquartile ranges [IQR]), as appropriate. Bold font indicates data with significant differences.

Table 2 Total Outcomes of Patients

\begin{tabular}{|l|c|}
\hline Outcome & Value \\
\hline Colistin MIC >2 (mg/L) & $0 / 210(0.0 \%)$ \\
I4-day mortality rate & $24(12.6 \%)$ \\
30-day mortality rate & $29(15.2 \%)$ \\
Median mortality time (days) & $9.0(6.0-12.8)$ \\
Duration of hospitalization, median (IQR), days & $34.0(21.0-56.8)$ \\
Bacteria elimination & $46(24.0 \%)$ \\
Time needed to clear bacteremia (days) & $8.0(6.0-13.0)$ \\
\hline
\end{tabular}

Notes: Data are reported as numbers (\%) or median values (interquartile ranges $[\mathrm{IQR}])$, as appropriate.

cumulative dose of PMB. After adjustments, use of mechanical ventilation (odds ratio $=3.043 ; 95 \%$ confidence interval: 1.253-7.389; $\mathrm{P}=0.014)$, vasoactive agents (2.560; 1.180-5.554; 0.017), multiple-site infection (1.280; $1.077-4.083 ; 0.001)$, and the total cumulative dose of PMB $(1.001 ; 1.000-1.001 ; 0.037)$ were independently associated

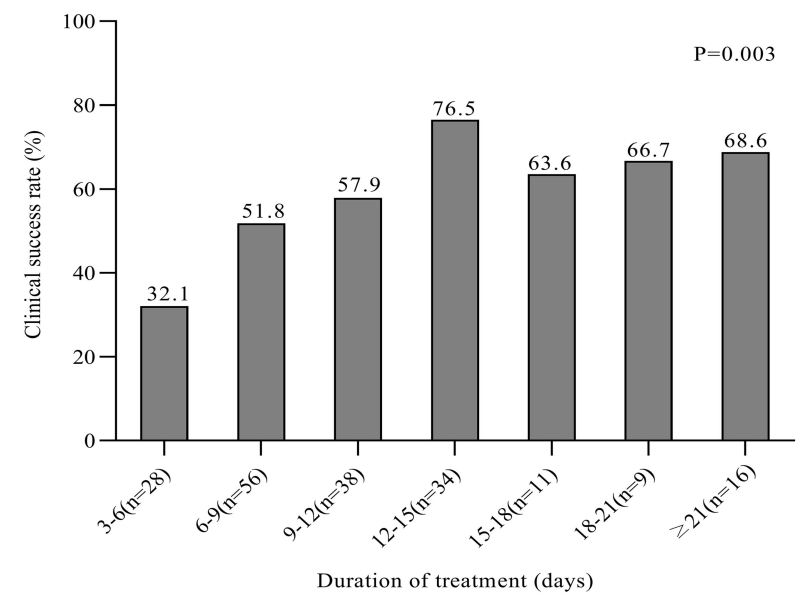

with the clinical efficacy of PMB (Table 3). Moreover, the results of Cox-regression survival analysis for 30-day mortality showed that the use of vasoactive agents (4.335; 1.006-18.685; 0.049) and the total cumulative dose of PMB (0.998; 0.996-0.999; 0.006) could influence survival time and mortality rate independently (Table 4). A high cumulative dose was an independent protective factor, while the use of vasoactive drugs was an independent risk factor (Figure 2). However, the cox regression survival analysis for 14-day mortality did not yield similar results (Table S2).

\section{Adverse Effects}

Overall, 22 cases $(11.5 \%)$ suffered the adverse effects of PMB treatment. The most prevalent adverse effect was nephrotoxicity $(\mathrm{n}, 8 ; 4.2 \%)$, followed by nerve-muscle blockade $(3 ; 1.6 \%)$ and skin hyperpigmentation (n, 3; $1.6 \%$ ). Moreover, some rare adverse effects occurred, including two cases of drug-induced fever, and drug-

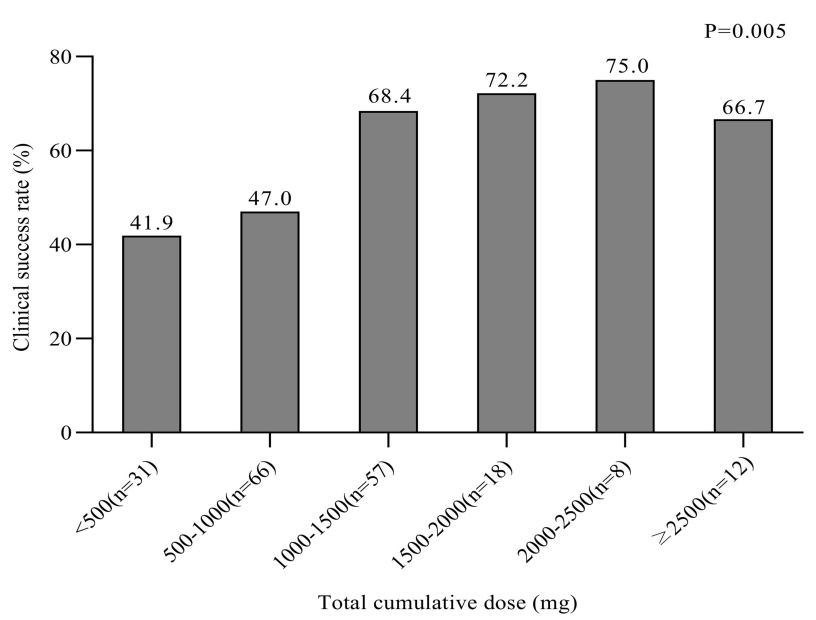

Figure I Prevalence of clinical success versus treatment duration and total cumulative dose (Mantel-Haenszel chi-square test). 
Table 3 Multivariate Logistic Regression of Clinical Efficacy of PMB Treatment

\begin{tabular}{|l|c|c|c|}
\hline Variable & Adjusted OR & $\mathbf{9 5 \%} \mathbf{~ C l}$ & P value \\
\hline Mechanical ventilation & 3.043 & $1.253-7.389$ & $\mathbf{0 . 0 1 4}$ \\
Vasoactive agents & 2.560 & $1.180-5.554$ & $\mathbf{0 . 0 1 7}$ \\
Multiple-site infection & 1.280 & $1.077-4.083$ & $\mathbf{0 . 0 0 1}$ \\
Total cumulative dose (mg) & 1.001 & $1.000-1.001$ & $\mathbf{0 . 0 3 7}$ \\
Treatment duration (days) & 0.960 & $0.906-1.018$ & 0.174 \\
\hline
\end{tabular}

Note: Bold font indicates data with significant differences.

Table 4 Cox-Regression Survival Analysis for 30-Day Mortality

\begin{tabular}{|l|c|c|c|}
\hline Variable & Hazard Ratio & $\mathbf{9 5 \%} \mathbf{~ I ~}$ & $\mathbf{P}$ \\
\hline Mechanical ventilation & $1.434 \times 10^{5}$ & $0.000-7.251 \times 10^{133}$ & 0.937 \\
Vasoactive agents & 4.335 & $1.006-18.685$ & $\mathbf{0 . 0 4 9}$ \\
Multiple-site infection & 1.907 & $0.873-4.165$ & 0.105 \\
Total cumulative dose (mg) & 0.998 & $0.996-0.999$ & $\mathbf{0 . 0 0 6}$ \\
Treatment duration (days) & 1.088 & $0.941-1.257$ & 0.253 \\
\hline
\end{tabular}

Note: Bold font indicates data with significant differences.

induced eruption, pruritus, nausea, general weakness, lethargy, and hepatotoxicity in one patient (Table 5).

\section{Discussion}

CRO infections (especially CRE) represent an urgent threat because few drugs are available to treat infections caused by these pathogens. PMB is one of the "last resort" choices for CRO infections. Therefore, the factors that affect PMB efficacy must be clarified for its rational and efficacious application.

We wished to explore the factors affecting PMB efficacy. We enrolled $191 \mathrm{CRO}$-infected patients; 110 of these patients had CS after PBM treatment and 81 had CF after PBM treatment. PMB was more efficacious in patients who had a longer duration of treatment and a higher total cumulative dose. There was a linear association between treatment duration and total cumulative dose that correlated with CS. More patients in the CF group had mechanical ventilation and vasoactive agents than those in the CS group.

The results of the current study are different from past investigations on the dose and efficacy of PMB. ${ }^{18-20}$ Elias et al showed (in a retrospective study) that increasing the daily dose of PMB ( $\geq 200 \mathrm{mg} /$ day) reduced in-hospital mortality, and that its benefits outweighed the risk of renal insufficiency. ${ }^{18}$ Mortality after PMB treatment was associated with an inappropriate daily dose $(<15,000$ units/ $\mathrm{kg} /$ day) in critically ill patients according to a retrospective study conducted by Ismail et al. ${ }^{19}$ An investigation on the association between the colistin dose taking into account body weight and microbiologic outcomes showed that higher doses led to better outcomes. ${ }^{20}$ However, the exact relationship between the daily dose and efficacy of
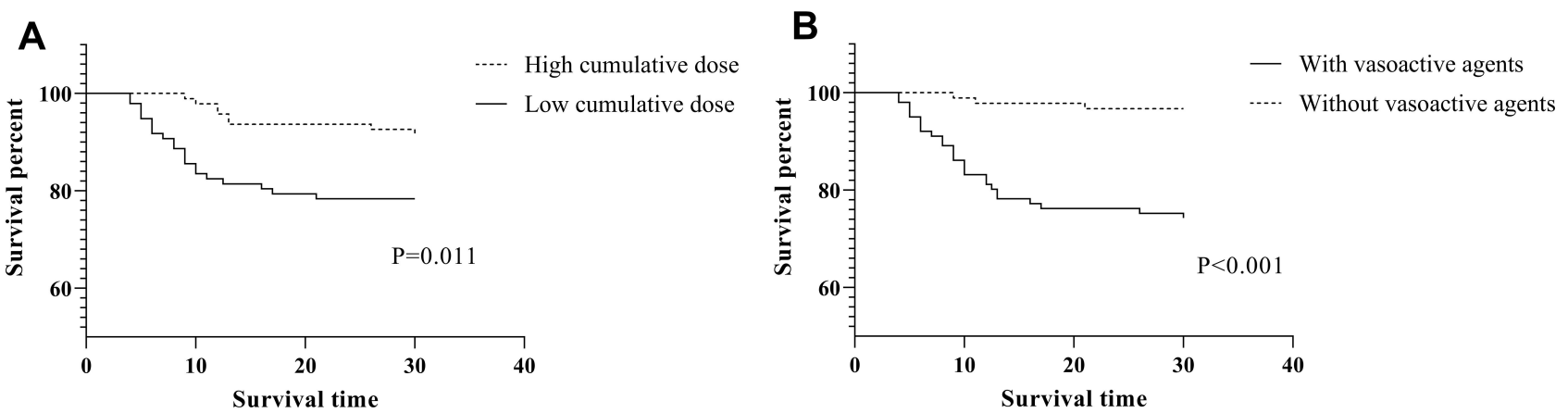

Figure 2 (A). Cox-regression survival analysis of cumulative dose for 30-day mortality. About half of the patients have a total cumulative dose of more than 1000 mg, defined as high cumulative dose. (B). Cox-regression survival analysis of vasoactive agents for 30-day mortality. 
Table 5 Adverse Reactions Following PMB Therapy

\begin{tabular}{|l|c|}
\hline Adverse Effects & Number (\%) \\
\hline Nephrotoxicity & $8(4.2 \%)$ \\
Nerve-muscle blockade & $3(1.6 \%)$ \\
Skin hyperpigmentation & $3(1.6 \%)$ \\
Drug-induced fever & $2(1.0 \%)$ \\
Drug-induced eruption & $\mathrm{I}(0.5 \%)$ \\
Pruritus & $\mathrm{I}(0.5 \%)$ \\
Nausea & $\mathrm{I}(0.5 \%)$ \\
General weakness & $\mathrm{I}(0.5 \%)$ \\
Lethargy & $\mathrm{I}(0.5 \%)$ \\
Hepatotoxicity & $\mathrm{I}(0.5 \%)$ \\
Total & $22(\mathrm{II} .5 \%)$ \\
\hline
\end{tabular}

PMB was not discovered in our study. In the International Consensus Guidelines for the Optimal Use of the Polymyxins published in $2019 .{ }^{17}$ The recommended daily dose of PMB is $1.25-1.50 \mathrm{mg} / \mathrm{kg}$ (q12h), and a loading dose of $2.0-2.5 \mathrm{mg} / \mathrm{kg}$ is suggested. However, the median daily dose of PMB in our study was $0.86 \mathrm{mg} / \mathrm{kg}$ (q12h). Furthermore, the recommended dose given by the PMB manufacturer was 500000-1000000 IU divided into two administrations per day, and the median daily dose in our study was 1000000 IU. Hence, the daily dose in our study was lower than that recommended in guidelines but in accordance with manufacturer's instructions. Obviously, the recommended dose in the manufacturer's instructions was lower than that in the guidelines. This different association of daily dose and efficacy may be attributed to the cutoff of $200 \mathrm{mg} /$ day set explicitly in Elias's study and the different evaluation methods for efficacy and in-hospital mortality. ${ }^{18}$ No scholars have reported on the relationship between the cumulative dose and efficacy of PMB. The total cumulative dose of PMB in patients in the CS group was higher than that in the CF group [1100.0 (700.01443.8) vs 800.0 (500.0-1112.5) $\mathrm{mg} ; \mathrm{P}=0.001]$. A high cumulative dose was an independent protective factor for 30-day mortality $(0.998 ; 0.996-0.999 ; 0.006)$, however, which was inevitably related to the longer duration of treatment.

Few studies have demonstrated that the efficacy of PMB is related to treatment duration. One retrospective study suggested that prolonging the duration of direct hemoperfusion with PMB-immobilized fibers can improve the clinical efficacy of PMB in septic-shock patients. ${ }^{21}$ No scholars have shown that mechanical ventilation and application of the vasoactive agents influence the efficacy of PMB. At the same time, the use of mechanical ventilation and vasoactive agents also inevitably means that patients have more serious diseases, which resulted in different baselines between CS and CF groups. However, PMB treatment has been shown to improve the prognosis of patients undergoing mechanical ventilation. ${ }^{22,23}$

Studies on the timing of medication administration have yielded differing results. In a study on the timing of PMB administration in CRKP-infected patients, early administration of PMB was found to be beneficial for improving bacterial clearance and patient survival. ${ }^{9}$ In our study, there was no statistical difference in the timing of PMB medication between different clinical effects. This may indicate that after the confirmation of CROs infection, the timing of PMB medication may not affect the clinical efficacy of PMB in the short term.

There was no difference in the proportion of common combination drugs between the CF and CS groups. It could be seen that the combination of drugs in this study may not be one of the factors that cause the difference in clinical efficacy. Several studies have also shown that PMB efficacy is related to its combination with other drugs such as tigecycline and enrofloxacin. ${ }^{8,24}$ The differences in these conclusions may be associated with the choice of endpoint indicators. PMB, tigecycline, and ceftazidime/avibactam are important drugs in the treatment of CRO infections. An in vitro study showed that CRE was much more sensitive to PMB than tigecycline, ${ }^{25}$ and that the efficacy of PMB and tigecycline combination was greater than that of PMB alone or tigecycline alone. ${ }^{11,26}$ The combination of PMB and ceftazidime has been demonstrated to have a synergistic effect on CRKP in vitro. ${ }^{27}$ However, a recent study had also shown that the combination of colistin and meropenem cannot produce clinical benefits, despite the synergistic effects in vitro. ${ }^{28}$

The prevalence of total adverse effects was $11.5 \%$ in our study. The prevalence of nephrotoxicity was $4.2 \%$, which is lower than that in the study by Mattos and colleagues $(40.5 \%),{ }^{29}$ Oliveira and co-workers $(26.8 \%),{ }^{30}$ and Agarwal and collaborators (11.8\%). ${ }^{6}$ PMB neurotoxicity is caused mainly by inhibition of acetylcholine in neuromuscular junctions and PMB reabsorption by renalcell receptors, but the overall mechanism is not known. ${ }^{31,32}$ The reason for the low prevalence of nephrotoxicity may have been the relatively low dose of PMB used in our study. The prevalence of skin hyperpigmentation was $1.6 \%$ in our study, which is lower than that reported by Mattos and colleagues $(8.1 \%) .{ }^{29}$ The reasons for pigmentation/darkening of skin vary, but the most 
common reason is melanin production in skin cells (especially in dermal macrophages). ${ }^{33}$

Our study had four main limitations. First, biases could not be controlled completely because this was a retrospective study and the baselines were not fully adjusted to match. Second, the objectivity of PMB efficacy as an endpoint was insufficient. Third, the study cohort was small. Finally, patients had various underlying diseases that would have affected PMB efficacy.

In the future, the factors that affect the efficacy and adverse reactions of PMB must be explored in depth. In addition, pharmacokinetic parameters should be investigated more deeply by measuring the drug concentration in the blood to achieve individualized administration of PMB.

\section{Conclusion}

PMB is an option for patients with CRO infection. It had good efficacy and a low prevalence of adverse reactions in the present study. The total cumulative dose and duration of PMB treatment, mechanical ventilation, vasoactive agents, and multiple-site infection were factors associated with the clinical efficacy of PMB.

\section{Abbreviations}

CROs, carbapenem-resistant organisms; PMB, polymyxin $\mathrm{B}$; CS, clinical success; CF, clinical failure; CREs, enterobacteriaceae-resistant organisms; MIC, minimum inhibitory concentration.

\section{Data Sharing Statement}

Available.

\section{Ethics Approval and Informed Consent}

The study protocol was approved by the Ethics Committees of the Second Xiangya Hospital of Central South University (LYF-2020021) in Changsha, China. This retrospective study involved patients admitted to the Second Xiangya Hospital of Central South University from 2018 to 2019.

\section{Consent for Publication}

Available.

\section{Acknowledgments}

We thank the supported grants of the National Scientific Foundation of China (No. 81503166), Hunan Provincial
Natural Science Foundation of China (2018JJ3718), WU JIEPING Medical Foundation (320.6750.19090-11), and the Hunan Provincial Department of Finance Grant (No.2019-93, No. 2018-92).

\section{Author Contributions}

JQ and QL conceptualized and designed the study. GHL, HHZ, YL and HYY acquired data. GHL, QQ and HY analyzed the data. GHL, HY, JQ, and QL drafted the manuscript. All authors made a significant contribution to the work reported, whether that is in the conception, study design, execution, acquisition of data, analysis and interpretation, or in all these areas; took part in drafting, revising or critically reviewing the article; gave final approval of the version to be published; have agreed on the journal to which the article has been submitted; and agree to be accountable for all aspects of the work.

\section{Funding}

This work was supported by the National Scientific Foundation of China (No. 81503166), Hunan Provincial Natural Science Foundation of China (2018JJ3718), WU JIEPING Medical Foundation (320.6750.19090-11), and the Hunan Provincial Department of Finance Grant (No.2019-93, No. 2018-92).

\section{Disclosure}

All authors report no conflicts of interest relevant to this article.

\section{References}

1. Chamieh A, El-Hajj G, Zmerli O, et al. Carbapenem resistant organisms: a 9-year surveillance and trends at Saint George University Medical Center. J Infect Public Health. 2019;13(12):2101-2106. doi:10.1016/j.jiph.2019.02.019

2. Fritzenwanker M, Imirzalioglu C, Herold S, et al. Treatment Options for Carbapenem- Resistant Gram-Negative Infections. Dtsch Arztebl Int. 2018;115(20-21):345-352. doi:10.3238/arztebl.2018.0345

3. Stansly PG, Schlosser ME. Studies on Polymyxin: isolation and Identification of Bacillus polymyxa and Differentiation of Polymyxin from Certain Known Antibiotics. J Bacteriol. 1947;54(5):549-556. doi:10.1128/JB.54.5.549-556.1947

4. Zavascki AP, Nation RL. Nephrotoxicity of Polymyxins: is There Any Difference between Colistimethate and Polymyxin B? Antimicrob Agents Chemother. 2017;61(3):e02319-16. doi:10.1128/AAC.0231916

5. Zakuan ZD, Suresh K. Rational use of intravenous polymyxin B and colistin: a review. Med J Malaysia. 2018;73(5):351-359.

6. Aggarwal R, Dewan A. Comparison of nephrotoxicity of Colistin with Polymyxin B administered in currently recommended doses: a prospective study. Ann Clin Microbiol Antimicrob. 2018;17(1):15. doi:10.1186/s12941-018-0262-0

7. Rigatto MH, Falci DR, Zavascki AP. Clinical Use of Polymyxin B. Adv Exp Med Biol. 2019;1145:197-218. 
8. Alves PH, Boff RT, Barth AL, et al. Synergy of polymyxin B, tigecycline and meropenem against carbapenem-resistant Enterobacter cloacae complex isolates. Diagn Microbiol Infect Dis. 2019;94(1):81-85. doi:10.1016/j.diagmicrobio.2018.11.011

9. Liang Q, Huang M, Xu Z. Early use of polymyxin B reduces the mortality of carbapenem-resistant Klebsiella pneumoniae bloodstream infection. Braz J Infect Dis. 2019;23(1):60-65. doi:10.1016/ j.bjid.2018.12.004

10. Dubrovskaya Y, Chen TY, Scipione MR, et al. Risk factors for treatment failure of polymyxin B monotherapy for carbapenem-resistant Klebsiella pneumoniae infections. Antimicrob Agents Chemother. 2013;57(11):5394-5397. doi:10.1128/ AAC.00510-13

11. Satlin MJ, Kubin CJ, Blumenthal JS, et al. Comparative effectiveness of aminoglycosides, polymyxin $\mathrm{B}$, and tigecycline for clearance of carbapenem-resistant Klebsiella pneumoniae from urine. Antimicrob Agents Chemother. 2011;55(12):5893-5899. doi:10.1128/AAC.00387-11

12. Chen Z, Liu W, Zhang Y, et al. Molecular epidemiology of carbapenem-resistant Acinetobacter spp. from XiangYa Hospital, in Hunan Province, China. J Basic Microbiol. 2013;53(2):121-127. doi: $10.1002 /$ jobm. 201100420

13. Pan S, Huang X, Wang Y, et al. Efficacy of intravenous plus intrathecal/intracerebral ventricle injection of polymyxin $\mathrm{B}$ for post-neurosurgical intracranial infections due to MDR/XDR Acinectobacter baumannii: a retrospective cohort study. Antimicrob Resist Infect Control. 2018;7(1):8. doi:10.1186/s13756-018-0305-5

14. Cai Y, Leck H, Tan RW, et al. Clinical Experience with High-Dose Polymyxin B against Carbapenem-Resistant Gram-Negative Bacterial Infections-A Cohort Study. Antibiotics. 2020;9(8):451. doi:10.3390/antibiotics9080451

15. Anania MC, Miranda C, Vizioli MG, et al. S100A11 overexpression contributes to the malignant phenotype of papillary thyroid carcinoma. J Clin Endocrinol Metab. 2013;98(10):E1591-600. doi:10.1210/jc.2013-1652

16. Dalyan CB, Topac T, Agca H, et al. Comparison of Clinical Laboratory Standards Institute (CLSI) and European Committee on Antimicrobial Susceptibility Testing (EUCAST) broth microdilution methods for determining the susceptibilities of Candida isolates. Mikrobiyol Bul. 2018;52(1):35-48. doi:10.5578/mb.63991

17. Tsuji BT, Pogue JM, Zavascki AP, et al. International Consensus Guidelines for the Optimal Use of the Polymyxins: endorsed by the American College of Clinical Pharmacy (ACCP), European Society of Clinical Microbiology and Infectious Diseases (ESCMID), Infectious Diseases Society of America (IDSA), International Society for Anti-infective Pharmacology (ISAP), Society of Critical Care Medicine (SCCM), and Society of Infectious Diseases Pharmacists (SIDP). Pharmacotherapy. 2019;39(1):10-39. doi:10.1002/phar.2209

18. Elias LS, Konzen D, Krebs JM, et al. The impact of polymyxin B dosage on in-hospital mortality of patients treated with this antibiotic. $J$ Antimicrob Chemother. 2010;65(10):2231-2237. doi:10.1093/jac/dkq285

19. Ismail B, Shafei MN, Harun A, et al. Predictors of polymyxin B treatment failure in Gram-negative healthcare-associated infections among critically ill patients. J Microbiol Immunol Infect. 2018;51 (6):763-769. doi:10.1016/j.jmii.2017.03.007

20. Vicari G, Bauer SR, Neuner EA, et al. Association between colistin dose and microbiologic outcomes in patients with multidrug-resistant gram-negative bacteremia. Clin Infect Dis. 2013;56(3):398-404. doi: $10.1093 / \mathrm{cid} / \mathrm{cis} 909$
21. Miyamoto K, Kawazoe Y, Kato S. Prolonged direct hemoperfusion using a polymyxin B immobilized fiber cartridge provides sustained circulatory stabilization in patients with septic shock: a retrospective observational before-after study. J Intensive Care. 2017;5(1):19. doi:10.1186/s40560-017-0214-3

22. Komatsu M, Hachiya T, Takahashi H, et al. Direct hemoperfusion with polymyxin B-immobilized fiber for the treatment of the acute exacerbation of idiopathic pulmonary fibrosis in patients requiring invasive mechanical ventilation. Respir Investig. 2017;55 (5):318-322. doi:10.1016/j.resinv.2017.05.001

23. Koami H, Sakamoto Y, Miyasho T, et al. The effects of polymyxin B-immobilized fiber hemoperfusion on respiratory impairment in endotoxemic pigs. $J$ Nippon Med Sch. 2014;81(3):130-138. doi: $10.1272 /$ jnms. 81.130

24. Guo W, Guo SC, Li M, et al. Successful treatment of extensively drug-resistant Acinetobacter baumannii ventriculitis with polymyxin B and tigecycline- a case report. Antimicrob Resist Infect Control. 2018;7:22. doi:10.1186/s13756-018-0313-5

25. Zhang R, Cai JC, Zhou HW, et al. Genotypic characterization and in vitro activities of tigecycline and polymyxin B for members of the Enterobacteriaceae with decreased susceptibility to carbapenems. $J$ Med Microbiol. 2011;60(Pt 12):1813-1819. doi:10.1099/ jmm.0.025668-0

26. Rao GG, Ly NS, Diep J, et al. Combinatorial pharmacodynamics of polymyxin B and tigecycline against heteroresistant Acinetobacter baumannii. Int $J$ Antimicrob Agents. 2016;48(3):331-336. doi:10.1016/j.ijantimicag.2016.06.006

27. Manning N, Balabanian G, Rose M, et al. Activity of Ceftazidime-Avibactam Against Clinical Isolates of Klebsiella pneumoniae, Including KPC-Carrying Isolates, Endemic to New York City. Microb Drug Resist. 2018;24(1):35-39. doi:10.1089/ mdr.2016.0293

28. Nutman A, Lellouche J, Temkin E, et al. Colistin plus meropenem for carbapenem-resistant Gram-negative infections: in vitro synergism is not associated with better clinical outcomes. Clin Microbiol Infect. 2020;26(9):1185-1191. doi:10.1016/j.cmi.2020.03.035

29. Mattos KPH, Gouvea IR, Quintanilha JCF, et al. Polymyxin B clinical outcomes: a prospective study of patients undergoing intravenous treatment. J Clin Pharm Ther. 2019;44(3):415-419. doi:10.1111/jcpt. 12801

30. Oliveira MS, Prado GV, Costa SF, et al. Polymyxin B and colistimethate are comparable as to efficacy and renal toxicity. Diagn Microbiol Infect Dis. 2009;65(4):431-434. doi:10.1016/j. diagmicrobio.2009.07.018

31. Duncan DA. Colistin toxicity. Neuromuscular and renal manifestations. Two cases treated by hemodialysis. Minn Med. 1973;56 (1):31-35.

32. Suzuki T, Yamaguchi H, Ogura J, et al. Megalin contributes to kidney accumulation and nephrotoxicity of colistin. Antimicrob Agents Chemother. 2013;57(12):6319-6324. doi:10.1128/AAC.00254-13

33. Dereure O. Drug-induced skin pigmentation. Epidemiology, diagnosis and treatment. Am J Clin Dermatol. 2001;2(4):253-262. doi:10.2165/00128071-200102040-00006 


\section{Publish your work in this journal}

Infection and Drug Resistance is an international, peer-reviewed openaccess journal that focuses on the optimal treatment of infection (bacterial, fungal and viral) and the development and institution of preventive strategies to minimize the development and spread of resistance. The journal is specifically concerned with the epidemiology of antibiotic resistance and the mechanisms of resistance development and diffusion in both hospitals and the community. The manuscript management system is completely online and includes a very quick and fair peerreview system, which is all easy to use. Visit http://www.dovepress.com/ testimonials.php to read real quotes from published authors. 